\title{
Managing sickness through COVID-19
}

\section{Claire Bennett}

Practice Management Consultant

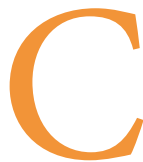

OVID-19 is having an unprecedented effect on dental practices throughout the UK and the working lives of the dental teams within them. One consequence has been increased rates of absenteeism due to sickness or self-isolation amongst staff.

Whilst routine dental care has been suspended during the delay phase of the pandemic, the usual issues associated with the management of high and prolonged sickness absence, for example, arranging temporary cover, ensuring services can continue and responding to any negative impact on staff morale, may themselves have been absent from operational priorities. However, it remains important for practices to communicate and adhere to existing relevant procedures and to understand how those procedures may have been affected by the current health crisis and recent legislative changes. Of course, it also remains vital that members of the dental team who are absent for COVID-19-related reasons are treated fairly and reasonably.

\section{Sickness and self-isolation}

Most practice owners understand that from time to time employees will become unwell and need to take some time off work. Most practices will have policies and procedures in place to manage such eventualities. However, in recent weeks, employers have been asked to follow the government's advice on self-isolation and shielding. Practice owners have had to get to grips quickly with what these terms mean and their impact on an individual's ability to take time off work for sickness and receive payment for that period.

The government's advice in these respects has changed several times since the beginning of the crisis. Currently, those with relevant symptoms, i.e. a persistent cough or high fever, are advised to self-isolate for 7 days, and longer where the fever persists. Individuals who do not have symptoms but live with someone who has a high temperature, or a new and continuous cough, must, together with their entire household, isolate for 14 days. People with specified serious underlying health conditions, who will have been written to by the NHS, should 'self-shield' for at least 12 weeks.

\section{Reporting procedures and evidence} Employees who are absent from work for a COVID-19 related reason should still be required to report their absence in accordance with normal sickness reporting procedures. Typically, that will involve notifying the practice as soon as possible that they are not fit for work; the reason they are not fit for work; and their likely date of return.

Employees can self-certify for the first 7 days of absence. This means they can 
confirm their incapacity for work without having to produce formal medical evidence. Most practices will require an employee to complete a self-certification form for this period.

If the absence continues beyond 7 days, employees can be required to provide medical evidence for their absence. Usually, this will take the form of a 'fit note'. Practices may need to be flexible in this respect for those absent from work due to COVID-19, as individuals are being advised not to attend health centres or hospitals. Instead, the government has introduced online isolation notes, which will confirm that the employee has been advised to self-isolate due to COVID-19, either because they have symptoms or live with someone who has symptoms. The notes can be accessed through the NHS website or NHS 111 online services and either be emailed to the individual or their employer directly at the former's request.

A letter from the NHS advising an employee to 'shield' because of a serious underlying health condition should also be treated as sufficient evidence of incapacity for work.

Managing the absence

Again, normal policies and procedures should be followed insofar as managing sickness absence due to COVID-19 is concerned. Regular contact with absent staff should be maintained to keep abreast of their medical condition and to inform them of any changes that might be taking place within the practice. What amounts to regular contact will vary from practice to practice, but could, for example, be a telephone call once a week or every fortnight.
It is important to keep full and accurate records of any communications between the practice and the employee, including the reason for their absence. File notes of telephone conversations and meetings should be made. Unsuccessful attempts to contact an employee should also be noted. Where action points or 'next steps' are agreed, it can be useful to summarise these in a letter to the employee. These records should be held confidentially.

\section{'Employees who are absent from work for a COVID-19 related reason should still be required to report their absence in accordance with normal sickness reporting procedures.'}

Whether absence for a COVID-19 related reason should count for the purposes of triggering disciplinary or capability procedures will depend on the circumstances. Generally, it is likely to be prudent to discount the absence and take no action. This should help mitigate the risk of employees coming into work when they should be selfisolating, thereby assisting the wider national effort to manage the virus. It should also help mitigate any litigation risk - the dismissal of an employee on a final written warning for absenteeism where they have been prevented from attended work due to COVID-19 is unlikely to find favour with an employment tribunal in most cases. It is also possible that discounting COVID-19 absences may amount to a reasonable adjustment for employees with disabilities.

Whilst most practices are largely closed, high numbers of absent staff due to sickness is unlikely to cause too many operational problems. As we move forward, this may change; with more practices requiring staff to be available for work at something like pre-COVID-19 levels. If high rates of sickness are a barrier to this, practices might explore with those absent due to a COVID-19 related reason, but who are feeling well enough, whether there is some work they can do from home.

Ordinarily, employees who meet the eligibility criteria are entitled to receive statutory sick pay (SSP) where they are absent from work for a period of at least 4 calendar days in a row and 3 'waiting days' have passed. In response to COVID-19, the SSP regime has been temporarily amended.

Qualifying employees who are selfisolating or shielding in accordance with government guidance and therefore are unable to work will be entitled to SSP. The usual 3 waiting days have been suspended, which means SSP is payable from day one of absence.

Other changes mean that employers with fewer than 250 employees, which will be most dental practices, will be reimbursed for the first 14 days of SSP due to COVID-19 related absence. These changes apply retrospectively with effect from 13 March 2020.

Of course, practices may pay employees contractual sick pay if that is a term of their contracts of employment.

\section{Return to work}

Given the current status of routine dentistry, an employee's return to work is unlikely to follow a familiar pattern. Nevertheless, it will continue to be important for practices to do all they reasonably can to support an employee's health, safety and wellbeing upon their return to work.

As part of this, practices should continue to carry out return to work meetings, whilst observing social distancing rules. Such meetings provide an opportunity for the employee to elaborate on the reason for their absence; confirm their fitness for work; and for the parties to decide whether any further action needs to be taken.

More generally, the current COVID-19 situation, will mean practices following public health guidance and NHS COVID-19 standard operating procedures, including, for example, those relating to good hand and respiratory hygiene, PPE and social distancing within the practice setting. Additional steps practices might take to ensure the health and safety of their team are to make sure managers know how to spot the signs of COVID-19 and what steps to take in response; keep the team updated on any actions being taken to reduce risks of exposure to them in the workplace; and, crucially, continue to communicate as the situation changes.

https://doi.org/10.1038/s41404-020-0413-9 